\title{
Modeling and Interpretation Wave Fields in Hierarchical Heterogeneous Medium
}

\author{
Olga A. Hachay ${ }^{1(\bowtie)}$, Andrey Y. Khachay ${ }^{2}$, and Oleg Y. Khachay ${ }^{2}$ \\ ${ }^{1}$ Institute of Geophysics UB RAS, Ekaterinburg, Russia \\ ${ }^{2}$ Ural Federal University, Ekaterinburg, Russia
}

\begin{abstract}
Geological medium is an open system which is influenced by outer and inner factors that can lead it to a unstable state. That non stability is as a rule occurred locally and these zones are named as dynamically active elements, which are indicators of potential catastrophic sources. These objects differ from the embedded geological medium by their structural forms, which often are of hierarchical type. The process of their activisation can be searched, using wave fields monitoring. For that purpose it is needed to develop new algorithms of modeling wave field's propagation through the local objects with hierarchical structure. Also it is needed to develop new theory of interpretation the wave field distribution for defining the contours of these local hierarchical objects. It had been constructed algorithms for 2D modeling of sound diffraction and linear polarized transversal seismic wave on an intrusion of hierarchic structure, located in the layer number $J$ of $\mathrm{N}$-layered elastic medium. We used the method of integral and integral-differential equations for a space frequency presentation of wave field's distribution. It is developed an algorithm for constructing the equation of theoretical inverse problem for 2-D electromagnetic field of $\mathrm{E}$ and $\mathrm{H}$ polarization and linear polarized longitudinal elastic wave by excitation of the $\mathrm{N}$-layered conductive or elastic medium with hierarchic conductive or elastic inclusion located in the $v$-th layer. From the theory it is obviously that for such complicated medium each wave field contains its own information about the inner structure of the hierarchical inclusion. Therefore it is needed to interpret the monitoring data for each wave field apart, and not to mix the data base. These results will be the base for constructing new systems of monitoring observations of dynamical geological systems. Especially it is needed to prevent rock shocks in deep mines by their exploitation.
\end{abstract}

Keywords: Hierarchic medium - Electromagnetic field - Seismic field • Algorithms of modeling - Equation of theoretical inverse problem

\section{Introduction}

The last decades are characterized by active development of Earth's sciences. We shall use the materials, published in the book (Dmitrievsky 2009) by Russian academician Dmitrievsky A.N., who suggested the conception about the development of oil-gas geology in Russian Federation. The modern research methods and technologies give the opportunity to obtain new data about the Earth's structure and processes, which 
occur in its interior. The conception development about the nonlinear geodynamics practically coincides with research of nonlinear processes in different parts of physics. In geology soliton and auto wave conceptions are developed, principles of synergetic and self organization become be used, and in geodynamics the macro quantum behavior of large mass matter, which are in critical state, in geophysics the auto wave nature of geophysical fields is researched. A new of direction quantum geodynamics appeared. In contrary from traditional approaches in geodynamics, which are based on classical models of continuum, quantum geodynamics allows analyzing the Earth's energetic structure, which evolutes in time and penetrates in all natural phenomena and possesses macro quantum time features. The development of possible methods and approaches must be based on the considering energy of dynamical processes.

For understanding and analyzing the state of the geological medium it is needed to compare the key ideas of geophysics, which give the main ideas to research different dynamical events into the Earth.

"Geophysics of XX century" is the understanding of such features:

- geophysical fields are indicators of the processes, which occur in lithosphere; geophysical parameters, which are registered distantly can have functional or correlation relation with the matter-structural characteristics of geological medium (on macro- and micro levels);

- analysis of space-time and energetic distribution of geophysical field can give information about space-time distribution of geological medium properties;

- registration and analysis of geophysical field in the monitoring regime can give information about the geodynamical processes in near borehole space, in Earth's crust and lithosphere in more deep Earth's layers.

Practical problems of geophysics of XX century had been vigorous stimulus of evolution of theoretical and experimental physics of thin layered, porous and crack media. As a result it had been derived new classes of mathematical models fluid saturated heterogeneous media, it had been researched anisotropic effects of geological media, and it had been revealed different physical and physical-chemical effects which occur on the boundaries "solid skeleton-fluid". Geophysics for the first time set a question about possibility of construction physical-geological and mathematical models of geological objects and processes. Applied geophysics of XX century had realized the possibility of research one and the same geological objects on micro level (nuclear geophysics), meso level (electrical, heat, magnetic, acoustic fields) and macro level (fields of elastic waves and low frequency electromagnetic fields).

Key ideas in XXI century in geophysics: Geophysics of the XXI century is the understanding of such features: the Earth is a self evolutionary, self conditioned geo cybernetic system for which the geophysical fields fulfill the role of a driving mechanism. Hierarchic and quantum features of geophysical space can be used in new geophysical principles together with nonlinear effects and effects of re-emission of geophysical fields that allow us to create a new geophysics. At the same time we have to consider new aspects in theory and mathematical modeling of geophysical fields and new systems of data interpretation. That will lead to development new equation classes, which describe the distribution of elastic and electromagnetic fields in heterogeneous and hierarchic media, with account of various effects of nonlinearity of geological 
media and irreversibility of geophysical processes. New theories of inverse problems solution with account of the hierarchic structure of inclusions imbedded into the layered medium will be developed.

Now we shall investigate and study some results which are into the key direction of geophysics of XXI century, noted by Dmitrievsky (Dmitrievsky 2009).

In present day for more adequate understanding of the dynamics of processes, which occur in the geological medium on deep levels by the action of natural and man caused factors Academician V.E. Panin using the results, which had been derived together with his colleagues (Panin 2005), had introduced a new paradigm on the junction between physics and mechanics of deformed solid body, which is the base of physical mesomechanics.

1. Identification of mechanisms of plastic flow on different structural deformation levels, which lead to a fundamental change of the initial inner structure of the solid body and forming into it dissipative substructures as mesoscopic plastic deformation support.

2. Fixing a relation between the outer action, changing of the initial inner structure, forming a hierarchy of mesoscopic self matched structural levels of deformation and occurring as a result of it mechanical fields.

3. Synergetic approach in the methodology of describing the deformed solid body as a non equilibrium many leveled medium, which in the points of bifurcation losses its shift stability on different structural levels and becomes to be destroyed in the conditions of global losses of its shift stability on macro scale level.

For experimental research of deformation mechanisms of specimens on meso level it had been developed new methods with use of speckle interferometer and optical television devices of technical vision, measuring of fractal dimension of deformed solid body. It turns out, that on the meso level 3-D structural elements (meso volumes) move as a whole. In that case it is sufficient to consider a representative volume, which consists on some tens meso volumes, for writing the equations of mesomechanics, taking into account the inner structure of the deforming solid body.

For realizing the second point of the new paradigm Panin with his colleagues had written a system of equations, which describes the mechanical field in the deforming solid body on one level (Panin 2005). It turned out, that it is similarly the Maxwell system of equations for alternating electromagnetic fields. Similarly electromagnetic field, where alternating electric and magnetic fields are mutually linked, the common mechanical field occurs in the deforming solid body, which contains organic mutual linked the translational and rotating modes (Panin 2005).

That result was very significant for choice of geophysical methods, using for monitoring of structure and state of rock massif, which is under strong man based action, which is a component of the geological medium in seismic-tectonically areas. 


\section{Mathematical Modeling and Comparison of Seismic and Electromagnetic Response for a Block-Layered 2D Model with Homogeneous Inclusions}

In that paper are derived integral equations and integral differential equations of $2 \mathrm{D}$ direct problem for the seismic field in the dynamical variant. We had been provided the joint analysis of the integral equations for $2 \mathrm{D}$ direct problems for electromagnetic and seismic fields. The received results can be used for definition of the complex criterions of achievement the research of high complicated medium both with seismic and electromagnetic methods. For the problem of sound diffraction on the 2D elastic heterogeneity, located in the $\mathrm{j}$-th layer of the n-layered medium, using the approach from V.I. Dmitriev and V.D. Kupradze (Hachay et al. 2013) we can derive the integral differential equation for the distribution of the potential for the vector of elastic displacements inside the heterogeneity.

$$
\begin{gathered}
\frac{\left(k_{1 j i}^{2}-k_{1 j}^{2}\right)}{2 \pi} \iint_{S c} \varphi(M) G_{S j}\left(M, M^{0}\right) d \tau_{M}+\frac{\sigma_{j a}}{\sigma_{j i}} \varphi^{0}\left(M^{0}\right) \\
-\frac{\left(\sigma_{j a}-\sigma_{j i}\right)}{\sigma_{j i} 2 \pi} \oint_{C} G_{S j} \frac{\partial \varphi}{\partial n} d c=\varphi\left(M^{0}\right) b y M^{0} \in S_{C} \\
\frac{\sigma_{j i}\left(k_{1 j i}^{2}-k_{1 j}^{2}\right)}{\sigma\left(M^{0}\right) 2 \pi} \int_{S c} \varphi(M) G_{S j}\left(M, M^{0}\right) d \tau_{M}+\varphi^{0}\left(M^{0}\right) \\
-\frac{\left(\sigma_{j a}-\sigma_{j i}\right)}{\sigma\left(M^{0}\right) 2 \pi} \oint_{C} G_{S j} \frac{\partial \varphi}{\partial n} d c=\varphi\left(M^{0}\right) b y M^{0} \notin S_{C}
\end{gathered}
$$

Using the second integral-differential presentation we can define the potential of the elastic displacements in the arbitrary layer, and then we can calculate the distribution of the vector of elastic displacements in the arbitrary layer. Let us compare the derived expressions with the solution of the diffraction problem for electromagnetic field in the frame of the same geometrical model. That case corresponds to the problem of exciting by a plane wave $\mathrm{H}$-polarization, the solution of which is done in the paper (Hachay et al. 2008a, b). Let us transform it to the form similarly to (1) and let us compare the derived equations for the solution of the inner 2-D seismic and electromagnetic problem. $\tilde{k}^{2}\left(M^{0}\right)=i \omega \tilde{\mu}_{0} \tilde{\sigma}\left(M^{0}\right) \tilde{\mu}_{0}=4 \pi 10^{-7} \frac{H}{m}, \tilde{\sigma}\left(M^{0}\right)$ - conductivity in the point $\mathrm{M}^{0}$, $\mathrm{i}$ - the imaginary unit, $H_{x}\left(M^{0}\right)$ - the summarized component of magnetic field, $H_{x}^{0}\left(M^{0}\right)$

- the component of magnetic field in the layered medium without heterogeneity, $\tilde{k}_{j i}^{2}\left(M^{0}\right)=i \omega \tilde{\mu}_{0} \tilde{\sigma}_{j i}, \tilde{k}_{i}^{2}\left(M^{0}\right)=i \omega \tilde{\mu}_{0} \tilde{\sigma}_{i}, \tilde{\sigma}_{j i}$ - conductivity into the heterogeneity, located into the j-the layer, $\tilde{\sigma}_{i}$ - conductivity of the i-th layer of the n-layered medium, $G_{m}\left(M, M^{0}\right)$ - the Green function of the 2-D problem for the case of H-polarization (Hachay 2007). 


$$
\begin{aligned}
& \frac{\left(k_{1 j i}^{2}-k_{1 j}^{2}\right)}{2 \pi} \iint_{S c} \varphi(M) G_{S j}\left(M, M^{0}\right) d \tau_{M}+\frac{\sigma_{j a}}{\sigma_{j i}} \varphi^{0}\left(M^{0}\right) \\
& -\frac{\left(\sigma_{j a}-\sigma_{j i}\right)}{\sigma_{j i} 2 \pi} \oint_{C} G_{S j} \frac{\partial \varphi}{\partial n} d c=\varphi\left(M^{0}\right) b y M^{0} \in S_{C} \\
& \frac{\left(\tilde{k}_{j i}^{2}-\tilde{k}_{j}^{2}\right)}{2 \pi} \iint_{S c} H_{x}(M) G_{m}\left(M, M^{0}\right) d \tau_{M}+\frac{\tilde{k}_{j i}^{2}}{\tilde{k}_{j}^{2}} H_{x}^{0}\left(M^{0}\right) \\
& -\frac{\left(\tilde{k}_{j i}^{2}-\tilde{k}_{j}^{2}\right)}{\tilde{k}_{j}^{2} 2 \pi} \oint_{C} H_{x} \frac{\partial G_{m}}{\partial n} d c=H_{x}\left(M^{0}\right) b y M^{0} \in S_{C}
\end{aligned}
$$

The difference in the boundary conditions for the seismic and electromagnetic problems lead to different types of equations: in the seismic case- to the integral-differential equation, in the electromagnetic case to the load integral Fredholm equation of the second type. If for the solutions of the direct electromagnetic and seismic in dynamical variant problems we can establish the similarity in the explicit expressions for the components of electromagnetic and seismic fields by definite types of excitation then with complicating of the medium structure as can we see from the obtained result by the case of the seismic field linked with longitudinal waves the similarity vanishes. That means that the seismic information is additional to the electromagnetic information about the structure and state of the medium.

For the problem of diffraction of a linearly polarized elastic transverse wave on the 2-D heterogeneity located in the $\mathrm{j}$-th layer of the n-layered medium, using the approach described in the paper (Hachay et al. 2008a, b) for the electromagnetic wave 2-D problem (case H-polarization), (the geometric model is similar to a that described higher in the previous problem) we obtain the expressions as follows for the components of the displacement vector:

$$
\begin{aligned}
& \frac{\left(k_{2 j i}^{2}-k_{2 j}^{2}\right)}{2 \pi} \iint_{S c} u_{x}(M) G_{S s, j}\left(M, M^{0}\right) d \tau_{M}+\frac{\mu_{j a}}{\mu_{j i}} u_{x}^{0}\left(M^{0}\right) \\
& -\frac{\left(\mu_{j a}-\mu_{j j}\right)}{\mu_{j i} 2 \pi} \oint_{C} u_{x}(M) \frac{\partial G_{S, j}}{\partial n} d c=u_{x}\left(M^{0}\right) b y M^{0} \in S_{C} \\
& \frac{\mu_{j i}\left(k_{2 j i}^{2}-k_{2 j}^{2}\right)}{\mu\left(M^{0}\right) 2 \pi} \iint_{S c} u_{x}(M) G_{S s, j}\left(M, M^{0}\right) d \tau_{M}+u_{x}^{0}\left(M^{0}\right) \\
& -\frac{\left(\mu_{j a}-\mu_{j i}\right)}{\mu\left(M^{0}\right) 2 \pi} \oint_{C} u_{x}(M) \frac{\partial G_{S, j}}{\partial n} d c=u_{x}\left(M^{0}\right) \text { by } M^{0} \in S_{C}
\end{aligned}
$$

The expressions (3) content the algorithm of seismic field simulation for distribution of transversal waves in the n-layered medium, which contain a $2 \mathrm{D}$ heterogeneity. The first expression is a Fredholm load integral equation of the second type the solution of which gives the distribution of the components of the elastic displacements vector inside the heterogeneity. The second of them is an integral expression for calculation of the elastic displacements vector in the arbitrary layer of the n-layered medium. Comparing the expressions (3) with correspondingly for the electromagnetic field (H-polarization) (2) we see that there is a similarity of the integral structure of these expressions. The difference is only for the coefficients of corresponding terms in the expressions (2) and (3). That we can account by choosing the system of observation with one or another field. We must also account the difference of the medium response 
frequency dependence from seismic or electromagnetic excitation. But keeping within the similarity of the coefficients the seismic field, excited by transversal waves, and the electromagnetic field will contain the similar information about the structure of the heterogeneous medium and state, linked with it. Those results are confirmed by the natural experiments described in the paper (Hachay 2007) Thus, it is showed that for more complicated, than horizontal-layered structures of the geological medium the similarity between the electromagnetic and seismic problems for longitudinal waves get broken. Therefore, these observations with two fields allow getting reciprocally additional information about the structure and especially about the state of the medium. These fields will differently reflect the peculiarities of the heterogeneous structures and response on the changing their state. If we can arrange seismic observations only with the transversal waves together with the magnetic component of electromagnetic one (H-polarization) in the 2-D medium, it will be establish the similarity, which can be used by construction of mutual systems of observation for magneto-telluric soundings and deep seismic soundings on exchanged waves.

\section{Mathematical Modeling of Seismic Response for a Hierarchic Model}

From the point of view of the paradigm of physical mesomechanics, which includes the synergetic approach to the change of rock massif state of different matter content, that problem can be solved with use of monitoring methods, which are settled on the research of hierarchic structured media (Panin 1995). For description of these effects it is needed to consider the wave process in the hierarchic blocked medium. Let us consider an algorithm of sound diffraction on 2-D elastic heterogeneity with hierarchic structure, located in the $\mathrm{j}$-th layer of n-layered medium (Hachay et al. 2008a, b).

$$
\begin{aligned}
& \frac{\left(k_{1 j i l}^{2}-k_{1 j}^{2}\right)}{2 \pi} \int_{S c l} \varphi_{l}(M) G_{S p, j}\left(M, M^{0}\right) d \tau_{M l}+\frac{\sigma_{j a}}{\sigma_{j i l}} \varphi_{l-1}^{0}\left(M^{0}\right) \\
& -\frac{\left(\sigma_{j a}-\sigma_{j i l}\right)}{\sigma_{j i l} 2 \pi} \oint_{C l} G_{S p, j} \frac{\partial \varphi_{l}}{\partial n} d c_{l}=\varphi_{l}\left(M^{0}\right) b y M^{0} \in S_{C l} \\
& \frac{\sigma_{j i l}\left(k_{1 j i}^{2}-k_{1 j}^{2}\right)}{\sigma\left(M^{0}\right) 2 \pi} \int_{S c l} \varphi_{l}(M) G_{S p, j}\left(M, M^{0}\right) d \tau_{M l}+\varphi_{l-1}^{0}\left(M^{0}\right) \\
& -\frac{\left(\sigma_{j a}-\sigma_{j i l}\right)}{\sigma\left(M^{0}\right) 2 \pi} \oint_{C l} G_{S p, j} \frac{\partial \varphi_{l}}{\partial n} d c_{l}=\varphi_{l}\left(M^{0}\right) b y M^{0} \notin S_{C l}
\end{aligned}
$$

$G_{S p, j}\left(M, M^{0}\right)$ source function for the longitudinal wave, which coincides with the function from the paper (Hachay and Khachay 2013), $k_{1 j i l}^{2}=\omega^{2}\left(\sigma_{j i l} / \lambda_{j i l}\right)-$ wave number for the longitudinal wave. In the equations, the index $j i$ indicates the features within the heterogeneity membership, $j a$ - out of the heterogeneity, $l=1 \ldots L-1-$ number of the hierarchic level, $\vec{u}_{l}=\operatorname{grad} \varphi_{l}, \varphi_{l}^{0}$ - potential of the normal seismic field in the layered medium, when the heterogeneity of the previous rank is absent. If $l=2 \ldots L \varphi_{l}^{0}=\varphi_{l-1}$, if $l=1, \varphi_{l}^{0}=\varphi^{0}$, this coincides with the expression from the paper (Hachay et al. 2015). 


$$
\begin{aligned}
& \frac{\left(k_{2 j i l}^{2}-k_{2 j}^{2}\right)}{2 \pi} \int_{S c l} u_{x l}(M) G_{S s, j}\left(M, M^{0}\right) d \tau_{M l}+\frac{\mu_{j a}}{\mu_{j l}} u_{x(l-1)}^{0}\left(M^{0}\right) \\
& -\frac{\left(\mu_{j a}-\mu_{j i l}\right)}{\mu_{j i} 2 \pi} \oint_{C l} u_{x l}(M) \frac{\partial G_{S, j}}{\partial n} d c_{l}=u_{x l}\left(M^{0}\right) b y M^{0} \in S_{C l} \\
& \frac{\mu_{j i l}\left(k_{2, j l}^{2}-k_{2 j}^{2}\right)}{\mu\left(M^{0}\right) 2 \pi} \int_{S c l} u_{x l}(M) G_{S s, j}\left(M, M^{0}\right) d \tau_{M l}+u_{x(l-1)}^{0}\left(M^{0}\right) \\
& -\frac{\left(\mu_{j a}-\mu_{j i l}\right)}{\mu\left(M^{0}\right) 2 \pi} \oint_{C l} u_{x l}(M) \frac{\partial G_{S, j}}{\partial n} d c_{l}=u_{x l}\left(M^{0}\right) b y M^{0} \in S_{C l}
\end{aligned}
$$

$k_{2 j i l}^{2}=\omega^{2}\left(\sigma_{j i l} / \mu_{j i l}\right)$ - wave number for the transversal elastic wave, $G_{S s, j}\left(M, M^{0}\right)$ source function for a transversal elastic wave (Hachay and Khachay 2013). If $l=2 \ldots$ $L u_{x}^{0}=u_{x(l-1)}$, if $l=1, u_{x l}^{0}=u_{x}^{0}$, this coincides with the expression from the paper (Hachay et al. 2015). If by transition on the next hierarchic level the axis of two-dimensionality does not change and only the geometry of the section of embedded structures change, then we can write the iteration process of modeling of the seismic field (case generation only longitudinal wave). The iteration process covers to modeling of the response of transition from the previous hierarchic level on the next level. Inside each hierarchic level the integral-differential equation and the integral-differential representation are calculated as it is written in the papers (Hachay et al. 2008a, b).

\section{Inverse Problem for Electromagnetic Field Propagation Through a Layered Conductive Medium with Hierarchic Inclusions}

In the paper (Hachay 1994) it had been considered a conception of a staged interpretation of the alternating electromagnetic field. On the first stage the parameters of the normal section or the parameters of the one dimensional non magnetic medium in which are embedded anomalous conductive or magnetic inclusions are defined. On the second stage it is developed a procedure of anomalous alternating field fitting by a system of singular sources, which are embedded into the horizontal layered medium with geoelectrical parameters, which had been defined on the first stage. On the third stage it is solved the theoretical inverse problem, that is for the given geoelectrical parameters for the embedded medium for the set of anomalous parameters, defined on the second stage, we define the contours of the inclusions. We had derived explicit integral-differential equations of the theoretical inverse problem for 2-D and 3-D alternating and 3-D stationary electromagnetic fields in a frame of the models: conductive or magnetic body in the J-th layer of the conductive layered half space. Here, using the approach, which was written in the papers (Hachay 1989, 1990), we had derived the equation of the theoretical inverse problem for the 2-D alternating electromagnetic field (scalar case) for the model: a conductive hierarchic inclusion of the $\mathrm{k}$ rank, located in the J-th layer of the conductive N-layered half space. $U^{+(k-1)}\left(M_{k}\right)$ anomaly component $E_{x}^{+(k-1)}$ or $H_{x}^{+(k-1)}$ for the inclusion of k-th rank by $M_{k} \in \partial D_{k}, U$ 
(M) - sum field $E_{x}$ or $H_{x}, U^{i(k-1)}(M)$ - normal field of the layered section $E_{x}$ or $H_{x}$ for $\mathrm{k}=1$, for $\mathrm{k}>1$ - the field $E_{x}$ or $H_{x}$, must be calculated, using the direct problem solution with use the algorithm (Hachay et al., 2013). $G^{a k}\left(M_{k}, M_{o}\right)$ - Green function for the inner area of the heterogeneity of the k-th rank, $G\left(M_{k}, M_{o}\right)$ - Green function of the $\mathrm{N}$-layered medium (Hachay 1989, 1990), $b_{v}, b_{i}, b_{a k}$ - complex coefficients for the J-th layer and for the inner area of the heterogeneity of the k-th rank, which are introduced in (3).

$$
\begin{aligned}
2 \pi U^{+(k-1)}\left(M_{0}\right)= & \int_{\partial D k}\left(\left(U_{v}^{+(k-1)}\left(M_{k}\right)\right.\right. \\
& \left.+U_{v}^{i(k-1)}\left(M_{k}\right)\right)\left(\frac{\partial G^{a k}\left(M_{k}, M_{0}\right)}{\partial n}-\left(\frac{b_{v}}{b_{i}}\right) \frac{\partial G\left(M_{k}, M_{0}\right)}{\partial n}\right) \\
& -b_{v}\left(\frac{\partial U_{v}^{+(k-1)}\left(M_{k}\right)}{\partial n}+\frac{\partial U_{v}^{(k-1)}\left(M_{k}\right)}{\partial n}\right) \\
& \left.\times\left(\left(\frac{1}{b_{a k}}\right) G^{a k}\left(M_{k}, M_{0}\right)-\left(\frac{1}{b_{i}}\right) G\left(M_{k}, M_{0}\right)\right)\right) d l_{k}
\end{aligned}
$$

The hierarchic heterogeneities are approximated by embedded non axial conductive $\sigma_{a k}$ cylinders, located along the axes OX. As a result of solution of the Eq. (1) for the function $r(\varphi)$, this describes the contour of the sought heterogeneity of the k-th rank.

\section{Conclusions}

We had developed an iterative algorithm for modeling electromagnetic and seismic fields in a frame of two models for layered-block 2D models with homogeneous and hierarchic inclusions. The last algorithms differ from the fractal model approach by a freer selecting of heterogeneities position of each rank. If the boundaries of the inclusion of the $\mathrm{k}$ rank are fractals (Mandelbrot 1982) the surface and contour integrals in the integral equations must be changed to repeated fractional integrals of Riman Liuvill (Simko et al. 1987). We had derived the equation of the theoretical inverse problem for the $2 \mathrm{D}$ alternating electromagnetic field (scalar case) for the model: a conductive hierarchic inclusion of the $\mathrm{k}$ rank, located in the J-th layer of the conductive N-layered half space.

\section{References}

Dmitrievsky, A.N.: Selected works, V.2, Nauka, Moscow (2009)

Hachay, O.A.: Geophysical monitoring of the state of rock massif with use of paradigm of physical mesomechanics. Phys. Earth 4, 58-64 (2007)

Hachay, O.A., et al.: Modeling of seismic and electromagnetic field in the hierarchic heterogeneous media. In: Proceedings of International Conference. IGF UB RAS, Ekaterinburg (2008a)

Hachay, O.A., et al.: Complex electromagnetic and seismic method of research of the crust and (2008b)

Hachay, O.A.: Mathematical modeling and interpretation of alternating electromagnetic field in heterogeneous crust and upper mantle of the earth. Professor Dissertation, IGF UB RAS, Sverdlovsk (1994) 
Hachay, O.A.: About interpretation 2-D alternative and 3-D stationary anomalies of electromagnetic field. Phys. Earth 10, 50-58 (1989)

Hachay, O.A.: About the solution of inverse problem of 3-D alternating electromagnetic fields. Phys. Earth 2, 55-59 (1990)

Mandelbrot, B.B.: The Fractal Geometry of Nature. Freeman, San Francisco (1982)

Panin, V.E.: Physical mesomechanics and computer construction of materials. Novosibirsk, Nauka, SB RAS (1995)

Simko, S.G., Kilbas, A.A., Marichev, O.I.: Integrals and derivatives of partial rank and some their applications. Minsk, Technique (1987) 